vated Mononuclear Leukocytes for Administration to Humans," as a part of its attempts to assist investigators in development of these products, and to provide a basis for establishing standards of safety and efficacy which are prerequisite for marketing approval. Perhaps more important, FDA has long publicized-and demonstrated-its willingness to meet with sponsors to discuss these and other issues. The design of clinical trials in oncology, as well as the appropriateness of various endpoint measurements, have been the subject of open and public debate in the oncology drug community. Trial designs and endpoints must be tailored to the particular disease entity and stage of disease under study and must take into account available therapy as well as the predicted effects of the test drug. FDA and its advisory committees have been active participants in discussions of these issues. During end-of-Phase I or Phase II conferences, FDA participants often use their expertise in these areas to evaluate a sponsor's proposals for clinical trials and to provide direct feedback on the suitability of the study design, including endpoints. It is particularty important that sponsors of novel and innovative therapies obtain FDA input into their plans.

The FDA has taken two positions that may have led to the impression that. the Agency has "discouraged" or "dissuaded" companies from filing applications for marketing LAK cells. First, as discussed above, FDA has indicated that the approval of a combination of new agents such as IL-2 and LAK cells would require a demonstration that both agents contribute to efficacy. Given the scientific evidence that LAK cells are very short-lived if infused without IL-2, FDA has not suggested that the activity of LAK cells alone be further investigated, but has consistently encouraged pharmaceutical companies and clinical investigators to determine whether LAK cells contribute to the efficacy of IL-2 plus LAK therapy.

Second, FDA has not specified the precise regulatory approach which would be used for activated lymphocyte therapies. Various LAK protocols represent just one subgroup of a broad and growing variety of cellular therapies being tested for a variety of indications. These raise a wide spectrum of scientific, legal, jurisdictional, public health, and resource allocation issues. Therefore, while policy alternatives have been under development and discussion at FDA for years, the regulatory approach will often need to be tailored to the specific case. When a sponsor is prepared to seek marketing approval for LAK cell or any other new cellular therapy, the
Agency is prepared to invoke appropriate regulatory mechanisms in a timely manner.

Finally, FDA's record during the past decade does not support McCormick's claim that "the regulatory apparatusschooled in specific and simples-is still not philosophically equipped for the complexities of treatment with BRMs." The licensing of alpha interferons, the immunosuppressive monoclonal antibody OKT3, and multivalent vaccines belies that assertion. However, we do agree with McCormick that "the object is to get an effective treatment to people who need it, "and that unnecessary regulatory delays most penalize patients waiting for new therapies. In fact, recent changes in FDA regulations should make investigational drugs available to patients who require them at an earlier time than previously and should shorten review times.

Janet Woodcock, M.D. Office of the Director Center for Biologics Evaluation and Research

Jay P. Siegel, M.D. Kathryn C. Zoon, Ph.D. Division of Cytokine Biology Center for Biologics Evaluation and Research

Henry I. Miller, M.D. A. Seth Pauker, Ph.D. Office of Biotechnology Office of the Commissioner Food and Drug Administration Rockville, MD 20857

\section{Cumon Daremext}

To the editor:

The editorial "Still a Few Bugs in the System" (Bio/Technology 8:981, Nov. '90) criticizes the final recommendations in the article "Are B.t.k. Plants Really Safe to Eat?" (same issue, p. 1011).

Transgenic plants are being engineered to express activated delta endotoxins from Bacillus thuringiensis var. kurstaki-not the biologically inactive protoxins that occur in insecticidal B.t.k. spore preparations. The author of the editorial, Douglas McCormick, believes that testing them as new agents before B.t.k. plants reach the dinner table would entail all too much bureaucratic delay. He argues that since B.t.k. spore preparations have been used safely as topical insecticides, $B$.t.k. plants should be marketed with little or no toxicological testing, and subsequently withdrawn if they turn out to be unsafe.

That argument flies in the face of current federal programs under which activated B.t.k. delta endotoxins, as expressed in plants, are likely to be regulated-those for pesticidal residues or food additives. Under the U.S. Food, Drug, and Cosmetic Act, the health risks associated with new pesticides and additives in food must be evaluated by federal regulators even if there is no $a$ priori indication that these chemicals pose a hazard. This is largely because many foods are widely consumed, so that a food-associated chemical that harms even a tiny proportion of consumers can harm large numbers of people.

McCormick denounces our recommendations as amounting "to clinical trials-for a food, mind you." Foods generally warrant a higher safety standard than drugs. Cancer patients, for example, may willingly tolerate the debilitating effects of chemotherapy drugs because such drugs are needed to increase their odds of survival. But most consumers would not tolerate side effects from food additives or pesticide residues; they do not need chemicals in their food. Moreover, clinical trials receive strict regulatory oversight be cause they are experiments on humans. Such human experimentation is precisely what we are trying to avoid by advocating that activated B.t.k. delta endotoxins be tested before they become part of the food supply.

The editorial also describes our recommended testing program as more extensive than it is. Most notably, we do not propose "studies in basic mammalian molecular biology to determine if B.t.k. toxin receptors-not yet firmly identified even in insects-are present in the human gut." Rather, we recommend relatively straightforward biochemical binding assays, in order to examine the affinity of mammalian gut cells for activated B.t.k. delta endotoxins.

In short, I believe our proposals are reasonable and consistent with longstanding and familiar policies for premarket approval of new chemicals added to food.

Rebecca J. Goldburg Scientist

Environmental Defense Fund 257 Park Avenue South New York, NY 10010

\section{A CORRECTION}

The photos in the article "Towards Understanding Human Genetic Diseases" (Bio/Technology 8:903, Oct. '90) were mislabeled. The larger (top) photo shows cells trisomic for chromosome 21 while the smaller (bottom) photo shows cells with the normal complement of two number 21 chromosomes. The caption had them reversed. 